K-C Chiu \& L Xu, (2003) "On generalized arbitrage pricing theory analysis: empirical investigation of the macroeconomics modulated independent state space model", 2003 International Conference on Computational Intelligence for Financial Engineering (CIFEr2003), pp. 139-144, March 20-23, Hong Kong.

\title{
On Generalized Arbitrage Pricing Theory Analysis: Empirical Investigation of the Macroeconomics Modulated Independent State-Space Model*
}

\author{
Kai-Chun Chiu and Lei Xu \\ Department of Computer Science and Engineering, The Chinese University of Hong Kong \\ Shatin, New Territories, Hong Kong, P. R. China \\ Emails: \{kcchiu,lxu\}@cse.cuhk.edu.hk
}

\begin{abstract}
Inception of Markowitz's modern portfolio theory has also fuelled the development of asset pricing models for empirical finance, ranging from linear single-factor models like the capital asset pricing model to fairly complex multi-factor models such as the arbitrage pricing theory $(A P T)$. It is well-known in the literature of finance that $A P T$ could be used for modelling the underlying security returns generation process. In this paper, we investigate a generalized version of the APT model, called the macroeconomics modulated independent state-space model, in terms of model specification adequacy as well as its performance on prediction. Empirical results reveal that the model is not only well-specified, but also superior to the temporal factor analysis model in stock price and index forecasting, thanks to its salient capabilities of modelling both short-term and long-term market dynamics.
\end{abstract}

Keywords: Arbitrage pricing theory, macroeconomic factors, white noise test, temporal factor analysis

\section{INTRODUCTION}

An important milestone of modern financial modelling was laid down by Markowitz's 1952 landmark paper [7] in modern portfolio theory. In the subsequent years, development in formal models of financial asset prices was witnessed by Sharpe's capital asset pricing model (CAPM) [13] in 1964, Merton's intertemporal CAPM [8] in 1973, Ross's arbitrage pricing theory (APT) [12] in 1976, and those of Lucas and Breeden. All such models have one thing in common. They are all based on a single notion of general equilibrium in which demand equals supply across all markets in an uncertain world where individuals and corporations act rationally to optimize their own welfare. It comes out of the result of interaction between prices, preferences and probabilities.

As uncertainties induce risk, one of the central questions of modern finance is the necessity of some tradeoff between risk and expected return. Defining the appropriate measures of risk and reward, determining how they might be linked through fundamental principles of economics and psychology, and then estimating such links empirically using historical data and performing proper statistical inference are

\footnotetext{
* The work described in this paper was fully supported by a grant from the Research Grant Council of the Hong Kong SAR (Project No: CUHK 4169/00E).
}

issues that rest on the heart of financial modelling for asset pricing.

Financial asset pricing models are usually divided over two fundamental issues. They are respectively what constitutes and how risk affects security returns. For instance, the CAPM model was structured on the belief that relevant risk measure is related to just one aspect of the macroeconomymarket fluctuation. Although inception of the model was largely attributable to the development of Markowitz's portfolio theory, a major breakthrough of the model was due to the identification and splitting between systematic and diversifiable idiosyncratic risk. Unfortunately, the model was not without its drawbacks. The most critical one was related to the assumptions underlying its derivation. The model was inextricably linked with quite a number of assumptions, and some of them, predominantly homogeneous expectation of investors about the market, were documented in the literature to be empirically unrealistic. To rectify this weaknesses as well as to further extend the capabilities of the CAPM model, the APT was proposed in [12], which assumed that the cross-sectional expected returns of securities follow a multi-factor model characterized by their sensitivities, usually called factor loadings. Both the CAPM and the APT models could be broadly regarded as typical representatives of the class of the more general, linear models. In fact, apart from modelling by linear regression, attempts have been made on nonlinear regression. The aim was to explore any substantially nonlinear hidden relationship. However, there are several disadvantage of nonlinear modelling as compared to the linear one. First, nonlinear modelling involves complicated procedures and it is not rare that the discovered optimum relation turns out to be counter-intuitive. Second, due to the diversity of all possible nonlinear relations, parametric modelling techniques become inappropriate and frequently it is difficult to find a suitable interpretation of the final results.

Previously, we have made an effort to solve the rotation indeterminacy problem encompassing the traditional APT by considering its temporal extension, called the temporal factor analysis (TFA) model. In this paper, we further investigate the macroeconomics modulated independent statespace model, which not only is an extension of the linear TFA model proposed in [16], but also has a salient feature of modelling both short-term and long-term dynamics in the financial market. As implied by its name, the model attempts to relate macroeconomic variables to the stock re- 
turns generation process. However, quite different from $[1,2,3,4,6,9,14]$ which explicitly modelled the relation between asset prices and real economic activities such as production rates, productivity, growth rate of gross national product, unemployment, yield spread, interest rates, inflation, dividend yields and so forth, it models such relation in a subtle way via the independent hidden factors that affect the stock market.

The rest of the paper is organized in the following way. Sections 2 briefly reviews the APT and the Gaussian TFA model respectively. Section 3 introduces, with considerable detail, the macroeconomics modulated independent statespace model. Section 4 investigates empirically its model specification adequacy. Section 5 compares its performance on stock indices prediction with the TFA model which is its degenerated variant. Section 6 concludes the paper.

\section{Review of Related Models}

This section briefly reviews two close ancestors of the macroeconomics modulated independent state-space model.

\subsection{The Arbitrage Pricing Theory}

The APT begins with the assumption that the $n \times 1$ vector of asset returns, $R_{t}$, is generated by a linear stochastic process with $k$ factors $[12,10,11]$ :

$$
R_{t}=\bar{R}+\mathbf{A} f_{t}+e_{t}
$$

where $f_{t}$ is the $k \times 1$ vector of realizations of $k$ common factors, $A$ is the $n \times k$ matrix of factor weights or loadings, and $e_{t}$ is a $n \times 1$ vector of asset-specific risks. It is assumed that $f_{t}$ and $e_{t}$ have zero expected values so that $\bar{R}$ is the $n \times 1$ vector of mean returns. The model addresses how expected returns behave in a market with no arbitrage opportunities and predicts that an asset's expected return is linearly related to the factor loadings or

$$
\bar{R}=R_{f}+\mathbf{A} p
$$

where $R_{f}$ is a $n \times 1$ vector of constants representing the riskfree return, and $p$ is $k \times 1$ vector of risk premiums. Similar to the derivation of CAPM, (2) is based on the rationale that unsystematic risk is diversifiable and therefore should have a zero price in the market with no arbitrage opportunities.

\subsection{Gaussian Temporal Factor Analysis}

Suppose the relationship between a state $y_{t} \in \mathbb{R}^{k}$ and an observation $x_{t} \in \mathbb{R}^{d}$ is described by the first-order statespace equations as follows $[15,16]$ :

$$
\begin{aligned}
& y_{t}=\mathbf{B} y_{t-1}+\varepsilon_{t}, \\
& x_{t}=\mathbf{A} y_{t}+e_{t}, \quad t=1,2, \ldots, N .
\end{aligned}
$$

where $\varepsilon_{t}$ and $e_{t}$ are mutually independent zero-mean white noises with $E\left(\varepsilon_{i} \varepsilon_{j}\right)=\Sigma_{\varepsilon} \delta_{i j}, E\left(e_{i} e_{j}\right)=\Sigma_{e} \delta_{i j}, E\left(\varepsilon_{i} e_{j}\right)=$
$0, \Sigma_{\varepsilon}$ and $\Sigma_{e}$ are diagonal matrices, and $\delta_{i j}$ is the Kronecker delta function:

$$
\delta_{i j}= \begin{cases}1, & \text { if } i=j, \\ 0, & \text { otherwise }\end{cases}
$$

We call $\varepsilon_{t}$ driving noise upon the fact that it drives the source process over time. Similarly, $e_{t}$ is called measurement noise because it happens to be there during measurement. The above model is generally referred to as the TFA model.

In the context of APT analysis, equation (1) can be obtained from equation (4) by substituting $\left(\tilde{R}_{t}-\bar{R}\right)$ for $x_{t}$ and $f_{t}$ for $y_{t}$. The only difference between the APT model and the TFA model is the added equation (3) for modelling temporal relation of each factor. The added equation represents the factor series $y=\left\{y_{t}\right\}_{t=1}^{T}$ in a multi-channel autoregressive process, driven by an i.i.d. noise series $\left\{\varepsilon_{t}\right\}_{t=1}^{T}$ that are independent of both $y_{t-1}$ and $e_{t}$. Specifically, it is assumed that $\varepsilon_{t}$ is Gaussian distributed. Moreover, TFA is defined such that the $k$ sources $y_{t}^{(1)}, y_{t}^{(2)}, \ldots, y_{t}^{(k)}$ in this state-space model are statistically independent.

\section{The Macroeconomics Modulated Independent State- Space Model}

\subsection{Overview of the Model}

The macroeconomics modulated independent state-space model was proposed by $\mathrm{Xu}$ with an aim to model the financial market in a state of general equilibrium. Unlike traditional APT, it further utilizes observed macroeconomic variables and indices to implement the concept of long-term equilibrium in economics. In general, the model takes the following form [16]:

$$
\begin{aligned}
y_{t} & =\mathbf{B} y_{t-1}+\mathbf{H} z_{t-1}+\varepsilon_{t} \\
x_{t} & =\mathbf{A} y_{t}+e_{t} \\
z_{t} & =\mathbf{C} y_{t}+\mathbf{E} v_{t}+\epsilon_{t}
\end{aligned}
$$

where $\varepsilon_{t}, e_{t}$ and $\epsilon_{t}$ are Gaussian white noises and independent from each other, $\varepsilon_{t}$ is independent of both $z_{t-1}$ and $y_{t-1}, e_{t}$ and $\epsilon_{t}$ are independent of $y_{t}$ and $v_{t}$.

Typically, $z_{t}$ consists of a number of macroeconomic indices and $v_{t}$ consists of a number of known non-market factors that affect the macroeconomy. Specifically, $\mathbf{H} z_{t-1}$ describes the indirect effect of the macroeconomic indices to the security market via the hidden factors $y_{t}$, and $\mathbf{C} y_{t}$ describes the feedback effect of the market to the macroeconomic indices.

We consider the model describes a capital market via both short-term and long-term dynamics. For short-term dynamics, $x_{t}, y_{t}$ and perhaps $z_{t}$ move to reach an equilibrium in the sense that the series of $\varepsilon_{t}, e_{t}$ and $\epsilon_{t}$ become stationary white noises, while the parameters $\mathbf{B}, \mathbf{H}, \mathbf{A}, \mathbf{C}, \mathbf{E}$ and the statistics of $\varepsilon_{t}, e_{t}$ and $\epsilon_{t}$ can be regarded as relatively constant due to slow changing. For long-term dynamics, the parameters $\mathbf{B}, \mathbf{H}, \mathbf{A}, \mathbf{C}, \mathbf{E}$ and the statistics of $\varepsilon_{t}, e_{t}$ and $\epsilon_{t}$ are all changing to cohere to equilibrium. 


\subsection{An Algorithm for Implementation}

An adaptive algorithm is given in [16] for implementation of the macroeconomics modulated independent state-space model. With $\mathbf{H}$ fixed, $\mathbf{H} z_{t}$ acts as a constant and can be regarded as a part of the mean of $\varepsilon_{t}$. The task of estimating $\mathbf{H}$ is a linear regression problem when $y_{t}, y_{t-1}$ and $\mathbf{B}$ are fixed. In particular, for $G\left(\varepsilon_{t} \mid \mathbf{H} z_{t-1}, \Lambda\right), G\left(e_{t} \mid 0, \boldsymbol{\Sigma}_{\mathbf{x}}\right)$ and $G\left(\epsilon_{t} \mid 0, \boldsymbol{\Sigma}_{\mathbf{z}}\right)$, the algorithm consists of four steps shown below.

Step 1 Estimate $y_{t}$ via $\hat{y}_{t}$ by maximum likelihood

$$
\begin{array}{r}
\hat{y}_{t}=\left[\boldsymbol{\Lambda}^{-1}+\mathbf{A}^{T} \boldsymbol{\Sigma}_{\mathbf{x}}{ }^{-1} \mathbf{A}+\mathbf{C}^{T} \boldsymbol{\Sigma}_{\mathbf{z}}{ }^{-1} \mathbf{C}\right]^{-1} \cdot\left[\mathbf{A}^{T} \boldsymbol{\Sigma}_{\mathbf{x}}{ }^{-1} \bar{x}_{t}\right. \\
\left.+\mathbf{C}^{T} \boldsymbol{\Sigma}_{\mathbf{z}}{ }^{-1}\left(\bar{z}_{t}-\mathbf{E} v_{t}\right)+\boldsymbol{\Lambda}^{-1}\left(\mathbf{B} y_{t-1}+\mathbf{H} \bar{z}_{t-1}\right)\right]
\end{array}
$$

Step 2 Update parameters of (6)

$$
\begin{aligned}
\mathbf{B}^{\text {new }} & =\mathbf{B}^{\text {old }}+\eta \operatorname{diag}\left[\varepsilon_{t} \hat{y}_{t-1}^{T}\right], \\
\mathbf{H}^{\text {new }} & =\mathbf{H}^{\text {old }}+\eta\left[\varepsilon_{t} \hat{z}_{t-1}^{T}\right], \\
\boldsymbol{\Lambda}^{\text {new }} & =(1-\eta) \boldsymbol{\Lambda}^{\text {old }}+\eta \operatorname{diag}\left[\varepsilon_{t} \varepsilon_{t}^{T}\right],
\end{aligned}
$$

Step 3 Update parameters of (7)

$$
\begin{aligned}
& \mathbf{A}^{\text {new }}=\mathbf{A}^{\text {old }}+\eta\left[e_{t} \hat{y}_{t}^{T}\right], \\
& \boldsymbol{\Sigma}_{\mathbf{x}}{ }^{\text {new }}=(1-\eta) \boldsymbol{\Sigma}_{\mathbf{x}}{ }^{\text {old }}+\eta \operatorname{diag}\left[e_{t} e_{t}^{T}\right],
\end{aligned}
$$

Step 4 Update parameters of (8)

$$
\begin{aligned}
\mathbf{C}^{\text {new }} & =\mathbf{C}^{\text {old }}+\eta\left[\epsilon_{t} \hat{y}_{t}^{T}\right], \\
\mathbf{E}^{\text {new }} & =\mathbf{E}^{\text {old }}+\eta\left[\epsilon_{t} \hat{v}_{t}^{T}\right], \\
\boldsymbol{\Sigma}_{\mathbf{z}}{ }^{\text {new }} & =(1-\eta) \boldsymbol{\Sigma}_{\mathbf{z}}{ }^{\text {old }}+\eta \operatorname{diag}\left[\epsilon_{t} \epsilon_{t}^{T}\right],
\end{aligned}
$$

In this paper, due to the absence of known non-market factors affecting the macroeconomy, the term $\mathbf{E} v_{t}$ is deleted by simply setting $\mathbf{E}=0$ in (8) and thus the relevant learning rules are omitted.

\section{White-Noise Test on Model Specification Adequacy}

It is common in the literature of statistics that test for model adequacy should immediately follow parameter estimation of the model under consideration. Usually a model is considered adequate only if the residual component consists of white noise. Since $\varepsilon_{t}$ in (6) is likely to be temporally correlated, we require the estimated residual to be substantially serially uncorrelated, i.e., autocorrelation of its lags should not be significantly different from zero. On the other hand, for both $e_{t}$ of (7) and $\epsilon_{t}$ of (8) to be adequate, the estimated residuals should be largely uncorrelated among its components.

\subsection{Data Consideration}

We carry out the analysis using past stock price and return data of Hong Kong. Daily closing prices of 30 actively trading stocks covering the period from January 1, 1998 to December 31, 1999 are used. The number of trading days throughout this period is 522. These stocks belong to the Hang Seng Index (HSI) constituents. HSI is the most representative index in the Hong Kong stock market. For macroeconomic indices, we use the 1 month Hong Kong Inter-Bank
Middle Rate, the Hang Seng Index and the Dow Jones Industrial Average (DJIA) respectively. The DJIA is used as a proxy in view of its significant co-integration effect [5] with the HSI.

\subsection{Data Preprocessing}

Before carrying out the analysis, the stock prices should be converted to stationary stock returns. The transformation applied can be described in four steps as shown below.

Step 1 Transform the raw prices to returns by

$$
R_{t}=\frac{p_{t}-p_{t-1}}{p_{t-1}} \text {. }
$$

Step 2 Calculate the mean return $\bar{R}$ by $\frac{1}{N} \sum_{t=1}^{N} R_{t}$.

Step 3 Subtract $\bar{R}$ from $R_{t}$ to get the zero-mean return.

Step 4 Let the result of above transformation be the adjusted return $\widetilde{R}_{t}$.

\subsection{Test Statistics}

To check if the residual $\varepsilon_{t}$ behave as a white-noise process, we adopt the Ljung-Box modified $Q$-statistic shown below. The $Q$-statistic can be used to test whether a group of autocorrelations is significantly different from zero.

$$
Q=N(N+2) \sum_{k=1}^{s} \frac{r_{k}^{2}(\hat{\varepsilon})}{N-k}
$$

where $N$ is the effective number of observations and $s$ is the lag order. If the sample value of $Q$ calculated above exceeds the critical value of $\chi^{2}$ with $s-1$ degrees of freedom at $\alpha=5 \%$, then we can conclude that at least one value of $r_{k}$ is statistically different from zero at $5 \%$ level of significance and suspect the residuals are serially correlated and not white.

On the other hand, to investigate whether each cross correlation coefficient of the observation noise residuals $e_{t}$ and $\epsilon_{t}$ is not significantly different from zero, we apply the $t$-test with the test statistic given by

$$
t=\frac{r}{\sqrt{1-r^{2}}} \cdot \sqrt{n-2},
$$

where $r$ is the correlation coefficient of a sample of $n$ points $\left(x_{i}, y_{i}\right)$ as given by

$$
r=\frac{\sum\left(x_{i}-\bar{x}\right)\left(y_{i}-\bar{y}\right)}{\left[\sum\left(x_{i}-\bar{x}\right)^{2} \sum\left(y_{i}-\bar{y}\right)^{2}\right]^{\frac{1}{2}}} .
$$

Assume that the $x$ and $y$ values originate from a bivariate Gaussian distribution, and that the relationship is linear, it can be shown that $t$ follows Student's $t$-distribution with $n-2$ degrees of freedom. Again the predefined level of significance is set at $\alpha=5 \%$.

\subsection{Empirical Test Results}

Results of $Q$ statistics and $p$-values for the residual $\varepsilon_{t}$ at lags from order 1 to 15 are shown in Table 1 . At $5 \%$ level of 
significance, autocorrelations of all residuals are not significantly different from zero. It implies that the specification of (6) is adequate. As for $e_{t}$, partial results showing $t$-test on correlation coefficients of the first two stocks with respect to the 30 HSI constituents are shown in Table 2. Results of the other 28 stocks are omitted due to space constraint. Out of 435 cross correlation coefficients, only 11 of them, or $2.53 \%$ are statistically significant at $\alpha=5 \%$. As the percentage is quite small, the results are satisfactory and we accept the null hypothesis that the residuals $e_{t}$ are substantially white. Similar results for $\epsilon_{t}$ are shown in Table 3 .

Table 1: Results showing $Q$-statistic and $p$-value of residual $\varepsilon_{t}$ for 30 HSI constituents.

\begin{tabular}{|c|c|c|c|c|}
\hline Lag & $Q$-Stat & $2 p$-value & $Q$ Q-Stat & $\overline{p \text {-value }}$ \\
\hline $\begin{array}{r}1 \\
2 \\
3 \\
4 \\
5 \\
6 \\
7 \\
8 \\
9 \\
10 \\
11 \\
12 \\
13 \\
14 \\
15\end{array}$ & $\begin{array}{r}\text { Resic } \\
0.0056 \\
0.4153 \\
1.8558 \\
2.6812 \\
4.3332 \\
6.0460 \\
7.8827 \\
7.8827 \\
8.0167 \\
8.7007 \\
9.1603 \\
9.2222 \\
11.6317 \\
13.3091 \\
13.3499\end{array}$ & $\begin{array}{l}\text { al } 1 \\
0.9403 \\
0.8125 \\
0.6029 \\
0.6125 \\
0.5025 \\
0.4181 \\
0.3431 \\
0.4450 \\
0.5325 \\
0.5607 \\
0.6071 \\
0.6838 \\
0.5581 \\
0.5024 \\
0.5753\end{array}$ & $\begin{array}{r}\text { Resic } \\
0.0077 \\
0.0081 \\
0.6221 \\
0.7548 \\
1.1613 \\
3.7385 \\
9.7960 \\
9.8682 \\
9.9039 \\
11.6716 \\
11.7318 \\
14.9980 \\
17.0208 \\
17.2598 \\
19.1754\end{array}$ & $\begin{array}{c}\text { al } 2 \\
0.9299 \\
0.9960 \\
0.8914 \\
0.9444 \\
0.9485 \\
0.7120 \\
0.2005 \\
0.2744 \\
0.3584 \\
0.3077 \\
0.3842 \\
0.2416 \\
0.1984 \\
0.2427 \\
0.2060\end{array}$ \\
\hline $\begin{array}{r}1 \\
2 \\
3 \\
4 \\
5 \\
6 \\
7 \\
8 \\
9 \\
10 \\
11 \\
12 \\
13 \\
14 \\
15\end{array}$ & $\begin{array}{l}\text { Resic } \\
0.0318 \\
0.2229 \\
0.2250 \\
0.4433 \\
0.5247 \\
3.0091 \\
4.3104 \\
4.3568 \\
5.0294 \\
5.2900 \\
5.3412 \\
6.1249 \\
7.6111 \\
7.6424 \\
7.8130\end{array}$ & $\begin{array}{l}\text { 1al } 3 \\
0.8585 \\
0.8945 \\
0.9735 \\
0.9788 \\
0.9912 \\
0.8077 \\
0.7434 \\
0.8236 \\
0.8317 \\
0.8710 \\
0.9135 \\
0.9096 \\
0.8680 \\
0.9071 \\
0.9310\end{array}$ & $\begin{array}{r}\text { Resic } \\
0.1188 \\
3.2264 \\
7.1968 \\
7.2105 \\
7.2607 \\
8.9177 \\
9.4983 \\
10.4737 \\
11.5217 \\
16.7004 \\
16.7067 \\
17.0780 \\
19.6578 \\
22.9557 \\
23.2691\end{array}$ & $\begin{array}{l}\mathrm{al} 4 \\
0.7303 \\
0.1993 \\
0.0659 \\
0.1252 \\
0.2020 \\
0.1783 \\
0.2189 \\
0.2334 \\
0.2417 \\
0.0813 \\
0.1169 \\
0.1468 \\
0.1041 \\
0.0611 \\
0.0787\end{array}$ \\
\hline
\end{tabular}

\section{Simulations of Stock Price and Index Prediction}

One possible application of the macroeconomics modulated independent state-space model is in stock price and index forecasting. Specifically, after setting up the model (6)-(8), we can use $x_{t}, z_{t}$ and $z_{t-1}$ to get $\hat{y}_{t}$. The predicted $\hat{x}_{t+1}$ can be obtained by $\mathbf{A} \hat{y}_{t+1}$ where $\hat{y}_{t+1}$ is approximately given by $\mathbf{B} \hat{y}_{t}+\mathbf{H} z_{t}$.

To explore the relative merit of modelling both short-term as well as long-term dynamics, we would compare the performance of the macroeconomics modulated independent state-space model with that of the TFA model. It is worthnoting that the TFA model is a degenerated variant of the
Table 2: Partial results of $t$-test on the residual $e_{t}$ for 30 HSI constituents. Only correlation coefficients the first two stocks with respect to 30 constituents are shown. Results of the other 28 stocks are omitted due to space constraint.

\begin{tabular}{rccc}
\hline \hline Stock \# & $\rho$ & $t$-stat. & $p$-value \\
\hline & & Stock \#1 \\
1 & 1.0000 & - & - \\
2 & -0.0704 & 1.6055 & 0.1090 \\
3 & 0.0710 & 1.6188 & 0.1061 \\
4 & -0.0120 & 0.2728 & 0.7851 \\
5 & -0.0111 & 0.2533 & 0.8001 \\
6 & 0.0587 & 1.3340 & 0.1828 \\
7 & -0.0143 & 0.3249 & 0.7454 \\
8 & 0.0417 & 0.9509 & 0.3421 \\
9 & -0.0074 & 0.1667 & 0.8677 \\
10 & 0.0255 & 0.5795 & 0.5625 \\
11 & 0.0491 & 1.1161 & 0.2649 \\
12 & 0.0076 & 0.1730 & 0.8627 \\
13 & -0.0270 & 0.6123 & 0.5406 \\
14 & 0.0023 & 0.0512 & 0.9592 \\
15 & -0.0316 & 0.7180 & 0.4731 \\
16 & -0.0318 & 0.7228 & 0.4701 \\
17 & -0.0244 & 0.5546 & 0.5794 \\
18 & -0.0836 & 1.9053 & 0.0573 \\
19 & -0.0687 & 1.5667 & 0.1178 \\
20 & 0.0090 & 0.2047 & 0.8379 \\
21 & 0.0641 & 1.4618 & 0.1444 \\
22 & -0.0472 & 1.0754 & 0.2827 \\
23 & -0.0480 & 1.0932 & 0.2748 \\
24 & 0.1017 & 2.3317 & 0.0201 \\
25 & 0.1057 & 2.4055 & 0.0165 \\
26 & 0.0205 & 0.4666 & 0.6410 \\
27 & -0.0451 & 1.0257 & 0.3055 \\
28 & 0.0817 & 1.8625 & 0.0631 \\
29 & 0.0457 & 1.0407 & 0.2985 \\
30 & 0.0299 & 0.6799 & 0.4969 \\
\hline & & &
\end{tabular}

macroeconomics modulated independent state-space model and could be obtained by setting $H=0$ in (6), thus ignoring the feedback of $z_{t-1}$ on $y_{t}$.

We demonstrate the results using the HSI and a representative stock, the Hong Kong and Shanghai Banking Corporation (HSBC) Holding. The HSBC holding is a constituent stock of the HSI and was ranked the first in market capitalization during the period. We use the first 400 data points for training and the remaining 120 data points for test. Both training and test are carried out in an adaptive fashion.

To illustrate stock index prediction, results by the TFA model and the macroeconomics modulated independent state-space model are shown in Figure 1 and 2 respectively.

Similarly, results for stock price prediction by the TFA model and the macroeconomics modulated independent state-space model are shown in Figure 3 and 4 respectively.

The performance of each model can be compared quantitatively by their respective root mean square errors (RMSE) between the predicted prices $\hat{p}_{t}$ and the desired prices $p_{t}$. As shown in Table 4, the macroeconomics modulated independent state-space approach consistently outperforms the TFA approach by having smaller RMSE for the HSI index and HSBC Holding. The results may be attributable to merit of modelling both short-term and long-term dynamics by the 
Table 2: Continued.

\begin{tabular}{rccr}
\hline Stock \# & $\rho$ & $t$-stat. & $p$-value \\
\hline & & & \\
1 & -0.0704 & 1.6055 & 0.1090 \\
2 & 1.0000 & - & - \\
3 & 0.0219 & 0.4988 & 0.6181 \\
4 & 0.0981 & 2.2356 & 0.0258 \\
5 & 0.0163 & 0.3699 & 0.7116 \\
6 & -0.0398 & 0.9031 & 0.3669 \\
7 & 0.0079 & 0.1781 & 0.8587 \\
8 & 0.0338 & 0.7689 & 0.4423 \\
9 & -0.0178 & 0.4049 & 0.6857 \\
10 & 0.0600 & 1.3648 & 0.1729 \\
11 & -0.0533 & 1.2140 & 0.2253 \\
12 & 0.0152 & 0.3460 & 0.7295 \\
13 & 0.0381 & 0.8662 & 0.3868 \\
14 & -0.0319 & 0.7277 & 0.4671 \\
15 & -0.0229 & 0.5216 & 0.6022 \\
16 & -0.0134 & 0.3052 & 0.7603 \\
17 & 0.0324 & 0.7391 & 0.4602 \\
18 & -0.0453 & 1.0321 & 0.3025 \\
19 & 0.0212 & 0.4833 & 0.6291 \\
20 & 0.0217 & 0.4946 & 0.6211 \\
21 & 0.0054 & 0.1226 & 0.9025 \\
22 & -0.0602 & 1.3709 & 0.1710 \\
23 & 0.0143 & 0.3249 & 0.7454 \\
24 & -0.0111 & 0.2521 & 0.8011 \\
25 & -0.0053 & 0.1200 & 0.9045 \\
26 & 0.0180 & 0.4076 & 0.6837 \\
27 & 0.1005 & 2.2973 & 0.0220 \\
28 & 0.0830 & 1.8976 & 0.0583 \\
29 & -0.1061 & 2.4262 & 0.0156 \\
30 & -0.0493 & 1.1231 & 0.2619 \\
\hline \hline & & & \\
\hline
\end{tabular}

Table 3: Results of $t$-test on the residual $\epsilon_{t}$ for 30 HSI constituents.

\begin{tabular}{rrrr}
\hline \hline Stock \# & corr. & $t$-stat. & $p$-value \\
\hline$\rho_{12}$ & -0.0205 & 0.4652 & 0.6420 \\
$\rho_{13}$ & -0.0052 & 0.1188 & 0.9055 \\
$\rho_{23}$ & 0.0498 & 1.1326 & 0.2579 \\
\hline \hline
\end{tabular}

macroeconomics modulated independent state-space model.

Table 4: Root mean square error (RMSE) for the two approaches

\begin{tabular}{lrr}
\hline \hline Model Type & HSI & HSBC \\
\hline $\begin{array}{l}\text { the TFA model } \\
\text { the macroeconomics modulated }\end{array}$ & 267.9210 & 1.5168 \\
independent state-space model & 218.8671 & 1.4745 \\
\hline \hline
\end{tabular}

\section{Conclusion}

In this paper, we empirically explore the macroeconomics modulated independent state-space model in two main aspects. First, we carry out white noise test to ensure model specification adequacy. Second, its performance in stock price and index forecasting is compared with that of the TFA model to illustrate the relative merit of simultaneously modelling both the short-term and long-term dynamics.

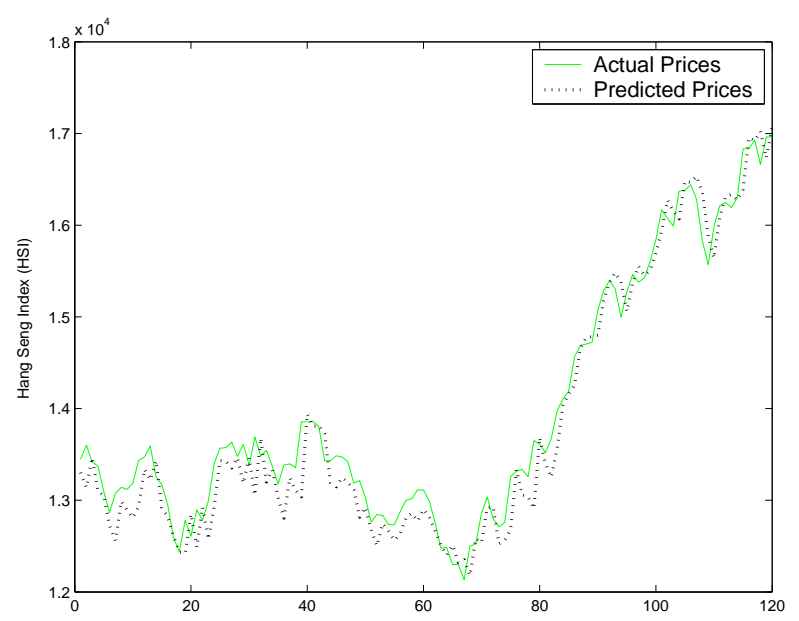

Figure 1: Predicted prices of HSI by the TFA model

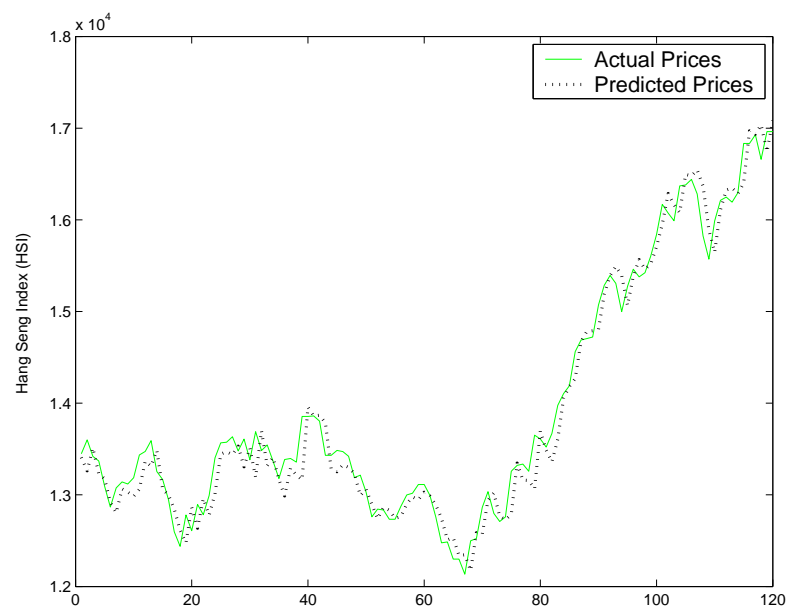

Figure 2: Predicted prices of HSI by the macroeconomics modulated independent state-space model

\section{REFERENCES}

[1] N. F. Chen. Financial investment opportunities and the macroeconomy. Journal of Finance, 46:529-554, 1991.

[2] N. F. Chen, R. Roll, and S. Ross. Economic forces and the stock market. Journal of Business, 59:383-403, 1986.

[3] S. K. Chung. Cointegration and causality between macroeconomic variables and stock market returns. Global Finance Journal, 10(1):71-81, 1999.

[4] E. F. Fama. Efficient capitial markets: Ii. Journal of Finance, 46:1575-1617, 1991.

[5] L. T. He. Time variation paths of international transmission of stock volatility - us vs. hong kong and south korea. Global Finance Journal, 12:79-93, 2001.

[6] R. D. Huang and W. A. Kracaw. Stock market returns and real activity: A note. Journal of Finance, 39:267273, 1984. 


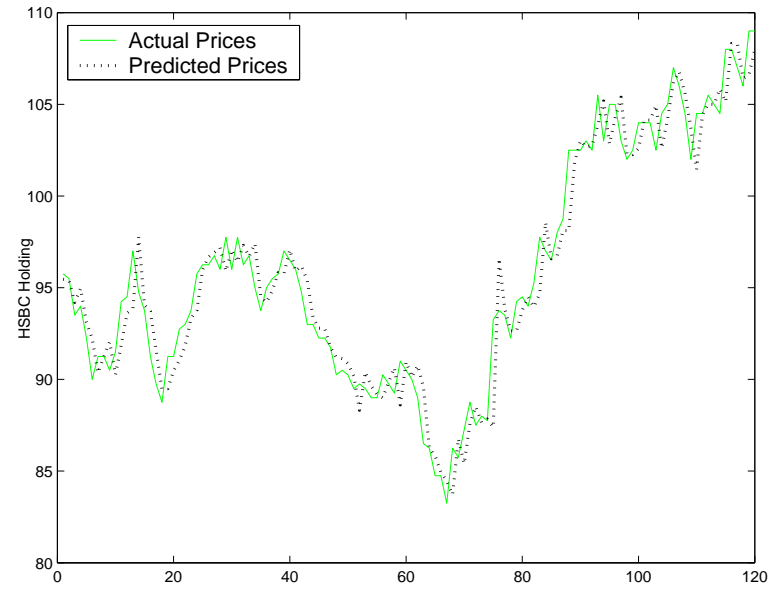

Figure 3: Predicted prices of HSBC Holding by the TFA model

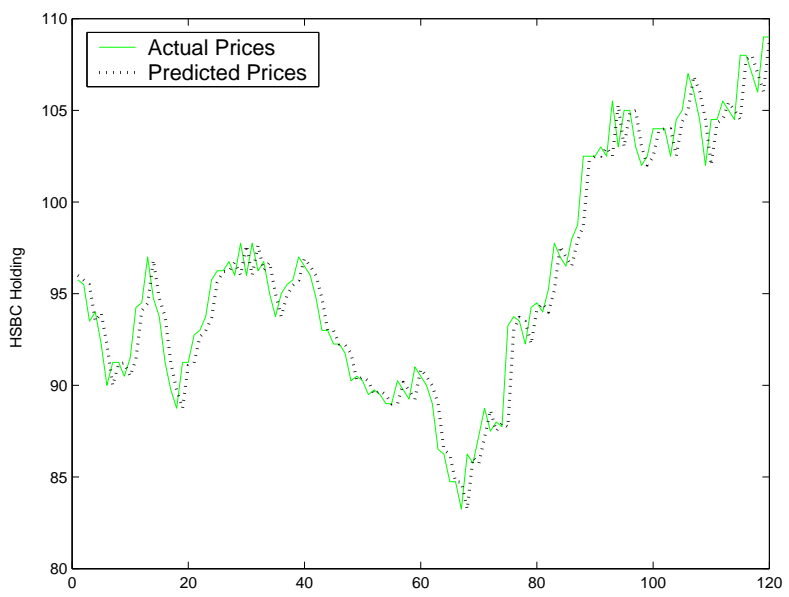

Figure 4: Predicted prices of HSBC Holding by the macroeconomics modulated independent state-space model

[7] H. Markowitz. Portfolio selection. Journal of Finance, 7:77-91, 1952.

[8] R. Merton. An intertemporal capital asset pricing model. Econometrica, 41:867-887, 1973.

[9] D. K. Pearce. Firm characteristics, unanticipated inflation, and stock returns. Journal of Finance, 43:965$981,1988$.

[10] R. Roll and S. Ross. An empirical investigation of the arbitrage pricing theory. Journal of Finance, 35:10731103, 1980.

[11] R. Roll and S. Ross. The arbitrage pricing theory approach to strategic portfolio planning. Financial Analysts Journal, 40:14-26, 1984.

[12] S. Ross. The arbitrage theory of capital asset pricing. Journal of Economic Theory, 13:341-360, 1976.

[13] W. F. Sharpe. Capital asset prices: A theory of market equilibrium under conditions of risk. Journal of Finance, 19:425-442, 1964.
[14] K. C. J. Wei and K. M. Wong. Tests of inflation and industry portfolio stock returns. Journal of Economics and Business, 44:77-94, 1992.

[15] L. Xu. Temporal byy learning for state space approach, hidden markov model and blind source separation. IEEE Trans. on Signal Processing, 48:21322144, 2000.

[16] L. Xu. Byy harmony learning, independent state space and generalized apt financial analyses. IEEE Transactions on Neural Networks, 12(4):822-849, 2001. 